\title{
Note on Relativistic Properties of Self-Energies
}

\author{
A. Pais and S. T. Epstein* \\ The Institute for Advanced Study, Princeton, New Jersey
}

\section{INTRODUCTION}

$I^{\mathrm{r}}$ $\mathrm{N}$ the recent developments in quantum field theory the question of the relativistic transformation properties of the self-energy of elementary particles plays an important role. As is well known, these self-energies, due to the interaction between particles and fields, are divergent quantities. While changing nothing in the fundamentals of the theory, an essential progress has been made, in first instance due to Schwinger, in that a set of "evaluation rules" is superposed on the formalism which makes it possible to recognize the self-energy of a free particle, i.e., a particle which is not acted on by an extraneous field, by its transformation properties of a simple inertial energy. This step forms part of a procedure to make an unambiguous separation between socalled "inertial" and "reactive" effects, aiming at a confrontation of the latter with experiment.

It is the aim of this note to draw attention to an aspect of relativistic theory which may perhaps be of some methodological use to obtain more insight into the meaning of the evaluation techniques mentioned above and of alternative prescriptions such as the application of "regulators" recently worked out by Pauli and his collaborators and described elsewhere in this issue.

The point we are driving at is: Let there be an energy momentum tensor density $T_{\mu \nu}$ characteristic for some physical system. If one observer moves in the $x$ direction with a constant velocity $v$ relative to another observer considered to be at rest, and our system is stationary (i.e., no explicit time dependence of its behavior is involved), then ${ }^{1}$

$$
\begin{gathered}
E=\frac{E(o)-v^{2} S(o)}{\left(1-v^{2}\right)^{\frac{1}{2}}}, \\
E=\int T^{4} \cdot{ }_{4} d v, \quad E(o)=\int T^{4} \cdot{ }_{4}(o) d v(o), \\
S(o)=\int T^{1} \cdot{ }_{1}(o) d v(o) .
\end{gathered}
$$

Here $E$ is the energy of the system from the point of view of the first observer, $E(o)$ the same quantity for the second observer. The quantity $S(o)$ we will call "stress." Equation (1) holds in classical theory as well as in quantum theory provided that in the latter case we con-

\footnotetext{
* Now at Columbia University, New York, New York.

1 The integrations are meant over, all of three-dimensional space. The index 1 refers to the $x$ direction. The light velocity is put equal to unity. Quantities marked $(o)$ refer to the rest system. Throughout the metrical tensor is taken to be $\delta_{i k}$.
}

sider $E, E(o), S(o)$ as expectation values referring to a specified state of the system.

On the basis of Dirac's positon theory, we now consider (1) for the case of a free electron of zero momentum. One should perhaps expect that, using the Schwinger or equivalent techniques of evaluating, the quantity $S(o)$ has necessarily to be zero, as these procedures give a mass-like behavior of the electromagnetic self-energy. One finds however that, up to the first order in $\alpha=e^{2} / \hbar$,

$$
S(o)=-(\alpha / 2 \pi) m,
$$

where $m$ is the mass of the electron. ${ }^{2}$

At first sight this may seem to be a disconcerting paradox which might lead one to doubt the consistency of the evaluation rules recently developed. Actually this seems to us not to be the case.

In fact, it is just the essential ambiguity inherent in handling divergent integrals which can make it on the one hand very possible to make a direct calculation of $E$ for a state with momentum $\mathbf{p}$ such that the way in which the contributions from various parts of momentum space are added depends itself on $\mathbf{p}$. One can say that the actual evaluation technique precisely picks out that $\mathbf{p}$ dependence which makes the energy as a function of $\mathbf{p}$ behave as if the stress were zero. On the other hand, the same prescription applied to the calculation of $S(o)$ where there is for that matter no $\mathbf{p}$ dependence at allneed then not necessarily give the result $S(o)=0 .{ }^{3}$ Thus, as long as one sticks to direct calculations of $E$ the question of what $S(o)$ is can simply not occur.

\section{THE DERIVATION OF $S(o)$}

The energy momentum tensor operator for electrons interacting with the electromagnetic field is

with

$$
T_{\mu \nu}=T_{\mu \nu}{ }^{\mathrm{mat}}+T_{\mu \nu}{ }^{\mathrm{elm}},
$$

Here

$$
T_{\mu \nu}^{\mathrm{mat}}=-\hbar / 4 i\left\{\bar{\psi} \gamma\left({ }_{\mu} \partial_{\nu)} \psi-\partial^{*}{ }_{(\mu} \bar{\psi} \cdot \gamma_{\nu)} \psi\right\}\right.
$$

$$
\bar{\psi}=i \psi^{*} \beta, \quad \partial_{\mu}=\partial / \partial x_{\mu}-i e \varphi_{\mu} .
$$

$\psi$ is the electron wave function, $\varphi_{\mu}$ the electromagnetic four-vector potential; $a_{(\mu} b_{\nu)}=a_{\mu} b_{\nu}+a_{\nu} b_{\mu}$. Further,

$$
T_{\mu \nu}{ }^{\mathrm{e}} \mathrm{lm}=-F_{\mu \rho} F_{\nu \rho}+\frac{1}{4} \delta_{\mu \nu} F_{\rho \sigma} F_{\rho \sigma},
$$

2 Whether considered as "mechanical" or renormalized to its experimental value makes in this order no difference due to the factor $\alpha$ in front.

3 If one calculates the energy for some value of $p$ by so-called non-covariant methods, one may, on the basis of (1) and (2), expect terms proportional to $\alpha p^{2}$ to remain in the expression for $E$. This actually happens in the calculations of N. M. Kroll and W. E. Lamb, Jr., Phys. Rev. 75, 388 (1949) and of J. B. French and V. F. Weisskopf, Phys. Rev. 75, 1240 (1949). 
where $F_{\rho \sigma}$ is the electromagnetic field. This last tensor has a zero trace. Therefore,

$$
T_{\mu \mu}=-\hbar / 2 i\left\{\bar{\psi} \gamma_{\mu} \partial_{\mu} \psi-\partial_{\mu}{ }^{*} \bar{\psi} \cdot \gamma_{\mu} \psi\right\} \text {. }
$$

In virtue of the Dirac equation,

$$
\left(\gamma_{\mu} \partial_{\mu}+m / \hbar\right) \psi=0, \quad \partial_{\mu}{ }^{*} \bar{\psi} \cdot \gamma_{\mu}-(m / \hbar \bar{\psi})=0,
$$

we have

$$
T_{\mu \mu}=m \psi^{*} \beta \psi
$$

Now, taking the expectation value for zero momentum, we have for symmetry reasons,

$$
\int T_{\mu \mu} d v=3 S(o)+E(o)=3 S(o)+m+\mu,
$$

where $\mu$ is the electromagnetic energy. This relation leads to a simple formula for $S(o)$ by means of the following consideration:

As the Hamiltonian $H$ of the total system involves $m$ explicitly only through the term $\beta m$ we have the operator relation $\beta=\partial H / \partial m$. Taking expectation values for the state under consideration one gets, therefore, $m \int \psi^{*} \beta \psi d v=m(1+\partial \mu / \partial m)$. Hence, from (3) and (4), ${ }^{4}$

$$
S(o)=-\frac{1}{3}[\mu-m(\partial \mu / \partial m)] \text {. }
$$

${ }^{4}$ The classical formula $S(o)=-\mu / 3$ can be considered as a special case of (5) as there $\mu$ does not depend on $m$. This leads to the well-known classical relation $E=\left(1-v^{2}\right)^{-\frac{1}{2}}\left\{m+\mu\left(1+v^{2} / 3\right)\right\}$. This deviation from a particle-like behavior has always been a difficulty in the days of classical electron theory. It is of course due to the existence of uncompensated forces in the system, and it is well known that for such "open" systems one generally has to expect $S(o)$ to be unequal to zero.

The case of the classical point electron provides an instructive example of the role of evaluation techniques. As was shown by L. L. Foldy and one of us, one can namely make a direct calculation of the energy in the sense mentioned above, using Fourier expansions of the fields in a $\mathbf{k}$ space, and proceeding in either of the following ways: (a) Adjoin to the energy contribution of a spherical shell between $k_{0}$ and $k_{0}+d k_{0}$ for an electron at rest, the contribu-
Using the well-known formula for $\mu$,

$$
\mu=3 \alpha / 2 \pi \cdot m[\log (\hbar / m a)+\text { const. }],
$$

where $a$ is an arbitrary cut-off length which is of course independent of $m$, and which eventually is supposed to tend to zero, one then obtains (1)..$^{5}$

In conclusion, we would like to emphasize once more that as long as one refrains from making explicit use of tensor transformation formulas of the type (1), the fact that $S(o) \neq 0$ can by the very nature of the evaluation techniques of Schwinger and Feynmann not cause any trouble whatsoever, as there only direct calculations of energies are performed. In other words, quite apart from whatever methods of evaluation or changes of the theory may be needed to make $S(o)$ to vanish, the present heuristic methods guarantee a particle like behaviour for calculations of the direct type. Thus the present form of quantum field theory is most suited to be adapted to whatever deeper going modifications of our notions may be necessary. There can be no doubt that a future theory, in which divergences do no longer occur in any physical statements, will either have $S(o)$ identically equal to zero or be such as to make meaningless any question as to the value of $S(o)$.

tion between the ellipsoidal shell between $k$ and $k+d k$, where $\mathbf{k}=\mathbf{k}_{0}+(\gamma-1) v^{-2}(\mathbf{k v}) \mathbf{v}, \gamma=\left(1-v^{2}\right)^{-\frac{1}{2}}$. This yields the above expression for $E$. (b) Choose for $\mathbf{k}$ the expression $\mathbf{k}=\mathbf{k}_{0}+(\gamma-1) v^{-2}\left(\mathbf{k}_{0} \mathbf{v}\right) \mathbf{v}$ $+\gamma k_{0} \mathbf{v}$. Now one gets for $E$ an expression from which the $v^{2} / 3$ term has vanished, i.e., now one has calculated as if $S(o)$ were zero. For $v=0$ both procedures coincide and give of course the above result for $S(o)$.

5 This result we also found some time ago by following Schwinger's method for computing the Lamb shift. In fact, one has only to replace $-j_{\mu} A_{\mu}^{\text {ext }}$ in the latter calculation by $\bar{\psi} \psi$ to get the formulas for the trace (3). The calculation is somewhat simplified by the fact that the terms of the Uehling type (vacuum polarization) are zero. We have further gone over to the special case $\mathbf{p}=0$ at an early stage. A better derivation has in the meantime been given by Villars to whose forthcoming paper, which also deals with the applications of regulators to this problem, we refer for further details. 